\title{
Associations and interactions among intestinal helminths of the brown trout, Salmo trutta, in northern Italy
}

\author{
B.S. Dezfuli ${ }^{1}$, L. Giari ${ }^{1}$, S. De Biaggi ${ }^{1}$ and R. Poulin ${ }^{2 *}$ \\ ${ }^{1}$ Dipartimento di Biologia, Università di Ferrara, Via Borsari 46, 44100 \\ Ferrara, Italy: ${ }^{2}$ Department of Zoology, University of Otago, PO Box \\ 56, Dunedin, New Zealand
}

\begin{abstract}
Species co-occurrences and interspecific associations between intensity of infection were studied in helminth communities of three populations of brown trout, Salmo trutta, from northern Italy. Of the eight helminth species, only four were common enough to be included in the analyses: Pomphorhynchus laevis, Acanthocephalus anguillae, Echinorhynchus truttae and Cyathocephalus truncatus. The observed frequencies of co-occurrences of the different species, based on presence/absence data, did not differ from those predicted by a null model derived from prevalence data. However, the intensity of infection (number of individuals per fish) of different helminth species were generally, but not always significantly, negatively correlated in tests of pairwise associations. Variation in fish sizes and its effect on infection levels, and whether or not two helminth species used the same or different intermediate hosts, had no influence on these findings. Of the few significant negative associations found between pairs of helminth species, none was found in more than one fish population. This suggests that interspecific associations may be conditiondependent: even in apparently similar localities, the same combinations of helminth species show different associations. Without evidence of replicability, it is almost impossible to conclude to the consistent role of competition between any pair of helminth species in the field.
\end{abstract}

\section{Introduction}

After years of investigation, there is still no agreement on whether or not interspecific interactions play an important role in structuring the helminth communities of vertebrate intestines (Poulin, 1998, 2001). Two lines of evidence have been pursued. First, a shift in niche or microhabitat preferences by one parasite species when it co-occurs with another species suggests that the two species are interacting. This can be inferred from field data (e.g. Bush \& Holmes, 1986; Ellis et al., 1999; VidalMartinez \& Kennedy, 2000) or, even better, it can be demonstrated using experimental infections (Holmes, 1961; Patrick, 1991). Second, the number of individuals of

*Author for correspondence

Fax: 643 479-7584

E-mail: robert.poulin@stonebow.otago.ac.nz one parasite species in one host may be dependent on the presence or numbers of a second species. These two effects are referred to by ecologists as functional and numerical responses, respectively (Thomson, 1980). In general, more weight is given to the demonstration of numerical responses when assessing the importance of interspecific interactions in community structure (Thomson, 1980).

Two approaches are commonly used to test for numerical effects in helminth communities. One is to verify whether the number of co-occurrences of different species differs from what would be expected by chance alone. This amounts to comparing observed frequencies of co-occurrence, based on presence/absence data only, with a null model generated from the actual prevalences of the different helminth species (Janovy et al., 1995). The second approach uses data on intensity of infection to 
look for significant positive or negative associations between pairs of helminth species across hosts (or infracommunities) in a sample. Some confounding factors can occasionally create spurious covariances between pairs of species (Lotz \& Font, 1994; Haukisalmi \& Henttonen, 1998; Poulin, 2001), but in general, consistent negative interactions are strong evidence of competitive interactions between species. Several biological processes can act to generate positive associations and mask any negative or competitive relationships. For instance, hosts such as fish tend to accumulate parasites over time, so that larger hosts often harbour more parasites than small ones (see Poulin, 2000). If this is true for different species of parasites, then they are likely to all occur in small numbers in small hosts, and in greater numbers in large hosts, potentially resulting in apparent positive associations among them when hosts in a sample vary in size. Also, since helminth larvae join an infracommunity of intestinal helminths when their intermediate host is ingested by the definitive host, there is the possibility that they arrive in packets with larvae of other helminth species (Bush et al., 1993). Helminth larvae of different species may be negatively or positively associated in their intermediate host (Lafferty et al., 2000), and these associations can be transferred to the definitive host (Lotz et al., 1995). It is thus important to consider the potential role of host size and shared intermediate hosts when studying patterns of species co-occurrence and associations among adult helminths in definitive hosts.

The brown trout, Salmo trutta, harbours a diverse parasite fauna within its native range (e.g. Kennedy, 1978). In the Province of Padua, northern Italy, brown trout is host to several intestinal helminth species, of which four are relatively common. Three of them, the acanthocephalans Pomphorhynchus laevis and Echinorhynchus truttae and the cestode Cyathocephalus truncatus, use the same intermediate host, the amphipod Echinogammarus stammeri (Dezfuli \& Scholz, 1995; Dezfuli et al., 1999). The fourth species, the acanthocephalan Acanthocephalus anguillae, uses the isopod Asellus aquaticus as intermediate host (Dezfuli et al., 1994). In the amphipod, $P$. laevis co-occurs more often than expected by chance with another but less common acanthocephalan of brown trout, Acanthocephalus clavula (Dezfuli et al., 2000). Cooccurrence patterns of $P$. laevis with either E. truttae or $C$. truncatus in the intermediate host could not be assessed, but if these species show significant associations in amphipods, they may also be associated in the fish host in ways that have nothing to do with competition or other forms of active interaction. Previous studies have examined interactions between some of the above helminths in other fish species, but their results seem highly dependent on the host species studied or on whether the study is experimental or based on natural infections (e.g. Bates \& Kennedy, 1990, 1991). Some aspects of community structure of helminths in brown trout are repeatable across localities (Kennedy \& Hartvigsen, 2000), but information on the consistency of pairwise species associations is lacking. It is therefore important to seek some sort of replication in studies of interspecific interactions among intestinal helminths, to assess their consistency across host populations.

The objectives of this study are to investigate the patterns of species co-occurrences and the correlations between numbers of different helminth species among hosts, in three replicate populations of the trout S. trutta. More specifically, we will determine whether any observed patterns can be explained in terms of: (i) variation in fish length within samples, or the simultaneous accumulation of different species by growing fish; and (ii) shared intermediate hosts, which can serve as a source of apparent associations. Excluding these two possibilities will strengthen any suggestion of interspecific interactions actually occurring in the fish host, provided that observed patterns are repeated across the three host populations.

\section{Materials and methods}

Fish were obtained from three streams (Tergola, San Giorgio and Grimana Nuova) from the Province of Padua, northern Italy. The streams are all tributaries of the Brenta river, and are of comparable depth, width and general physical characteristics. The main other fish species found in these streams are Oncorhynchus mykiss, Leuciscus cephalus, Barbus barbus, Scardinius erythrophthalmus and Gasterosteus aculeatus. The fish were captured by electric fishing between November 1998 and December 1999; small numbers of fish were obtained for each month and pooled by stream for analysis. This pooling was done because a preliminary analysis revealed that of 12 possible tests ( 3 streams times 4 helminth species) for seasonal changes in numbers of helminths per fish, only three were significant (Kruskal-Wallis tests, $P<0.05$ ), with variation among streams being much more substantial than seasonal variation within stream.

Each fish was anaesthetized following capture and then killed by a blow to the head. Its total length was recorded prior to dissection. The entire digestive tract of each fish was removed from the fish, fixed in $8 \%$ formaldehyde and opened subsequently in the laboratory. All helminths found in the intestine of each individual trout were identified and counted.

Fish from the three different streams were treated separately in the analyses. A comparison of fish sizes among the three streams was performed using an ANOVA. To determine whether co-occurrences of different helminth species in the same fish host were more or less frequent than expected by chance, we generated a null model of expected frequencies based on the actual prevalence of the different helminth species (see Janovy et al., 1995). In essence, the probability of any two or more species co-occurring in a fish is equal to the product of their prevalences in the sample. Observed and expected frequencies were compared with a Chi-square test.

Other analyses of species associations used the numbers of parasites of each species per fish. Since these numbers do not meet the assumptions of parametric tests, Spearman's rank correlation coefficients were used to test for relationships between fish length and either intensity of infection by each parasite species, total helminth abundance, or species richness (number of different helminth species per fish). Spearman's correlation coefficients were also used to assess all pairwise relationships between the intensity of two parasite species across fish hosts. These correlation analyses 
Table 1. Mean length (and range) of trout, Salmo trutta, from three streams in northern Italy, with summary statistics on infection by eight intestinal helminth species (prevalence and mean intensity of infection, with range of intensities in parentheses).

\begin{tabular}{lccc}
\hline & Tergola & San Giorgio & Grimana Nuova \\
\hline $\begin{array}{l}\text { No. fish examined } \\
\text { Total length, cm }\end{array}$ & 46 & 68 & 53 \\
& $27.6(21.5-40.5)$ & $26.3(15.8-53)$ & $19.8(10.7-30)$ \\
Acanthocephala & & & \\
$\quad$ Pomphorhynchus laevis & $28.3 \%, 4.4(1-24)$ & $75.0 \%, 33.1(1-248)$ & $11.3 \%, 1.5(1-3)$ \\
$\quad$ Acanthocephalus clavula & - & $4.4 \%, 1.7(1-2)$ & - \\
$\quad$ A. anguillae & $32.6 \%, 1.7(1-6)$ & $20.6 \%, 5.1(1-15)$ & $3.8 \%, 2.5(2-3)$ \\
$\quad \begin{array}{l}\text { A. lucii } \\
\text { Echinorhynchus truttae }\end{array}$ & $6.5 \%, 1.7(1-2)$ & - & - \\
$\quad$ Neoechinorhynchus rutili & $17.4 \%, 2.4(1-7)$ & $47.1 \%, 4.5(1-36)$ & $1.9 \%, 2.0(-)$ \\
$\quad-$ & $4.4 \%, 1.0(-)$ & - \\
Cestoda & - & $45.6 \%, 7.7(1-85)$ & $35.8 \%, 5.7(1-42)$ \\
$\quad$ Cyathocephalus truncatus & $67.4 \%, 4.8(1-41)$ & & - \\
Nematoda & & $4.4 \%, 5.0(1-13)$ & \\
$\quad$ Raphidascaris acus & $2.2 \%, 1.0(-)$ & & \\
\hline
\end{tabular}

excluded double-zeros, i.e. fish not infected by either parasite species in a pair; all correlation coefficients were corrected for tied ranks. A comparison of intensity of infection by each parasite species across the three streams was performed using a Kruskal-Wallis test.

\section{Results}

A total of 167 trout were obtained from the three streams. From these, a total of 2546 worms belonging to eight species were recovered: six acanthocephalan species, one cestode species and one nematode species (table 1). Only four helminth species were common enough to allow analyses of their co-occurrences and interspecific associations: Pomphorhynchus laevis, Acanthocephalus anguillae, Echinorhynchus truttae and Cyathocephalus truncatus. Their intensity of infection varied significantly among the three streams (Kruskal-Wallis test: $P$. laevis, $H=65.52, P=0.0001 ; A$. anguillae, $H=$ 12.25, $P=0.0022 ;$ E. truttae, $H=34.97, P=0.0001 ; C$. truncatus, $H=6.63, P=0.0364)$. The mean length of fish also varied among the three streams (ANOVA: $F_{2,164}=$ 37.403, $P=0.0001$ ), with fish from the Grimana Nuova stream being smaller than fish from the other two streams (table 1).

The observed frequencies of co-occurrence of the four main helminth species did not deviate significantly from those predicted by a null model based on random occurrences derived from prevalence data (fig. 1). There was a weak tendency for fewer fish harbouring single species than expected, and more uninfected fish than expected, but these were not statistically significant (fig. 1).

Numbers of E. truttae per fish were significantly correlated with fish length in one of the three streams; none of the other correlations proved to be significant at $\alpha=0.05$ (table 2). Of the 12 correlation coefficients computed, six were negative and six were positive. Fish length also did not correlate with either species richness, i.e. the number of different helminth species per fish, or total helminth abundance, in any of the three study streams (table 2).

Of the 17 pairwise correlations between the intensity of infection by two helminth species that could be computed, 16 were negative; of these, only four were statistically significant at $\alpha=0.05$ (table 3 ). The four significant correlations came from four different species pairs and two different streams. These results can also be seen from the perspective of shared or different intermediate hosts. Three out of eight pairwise associations involving two helminths with different intermediate hosts ( $A$. anguillae and another species) were significantly negative, whereas only one of the nine pairwise associations between helminths that share the same intermediate host was negative (table 3 ).

\section{Discussion}

Patterns of species associations in naturally infected hosts, both in terms of presence/absence data and in terms of actual intensity of infection, can be used as a first step toward elucidating the role of interspecific

Table 2. Relationships (Spearman's rank correlation coefficients) between total length of trout, Salmo trutta, and various parameters of infection: the number of worms per fish for four common helminth species, the total abundance of helminths per fish (all species combined), and the infracommunity species richness (number of parasite species per fish). Data are presented separately for three streams in northern Italy.

\begin{tabular}{lrcr}
\hline & Tergola & $\begin{array}{c}\text { San } \\
\text { Giorgio }\end{array}$ & $\begin{array}{c}\text { Grimana } \\
\text { Nuova }\end{array}$ \\
\hline Pomphorhynchus laevis & -0.118 & -0.037 & 0.224 \\
Acanthocephalus anguillae & 0.148 & -0.073 & 0.149 \\
Echinorhynchus truttae & 0.119 & $0.312^{* *}$ & -0.014 \\
Cyathocephalus truncatus & -0.073 & $0.214^{*}$ & -0.133 \\
Total abundance & -0.072 & 0.007 & -0.089 \\
Species richness & -0.028 & 0.157 & 0.039 \\
\hline
\end{tabular}

${ }^{*} P<0.10,{ }^{* *} P<0.05$. 

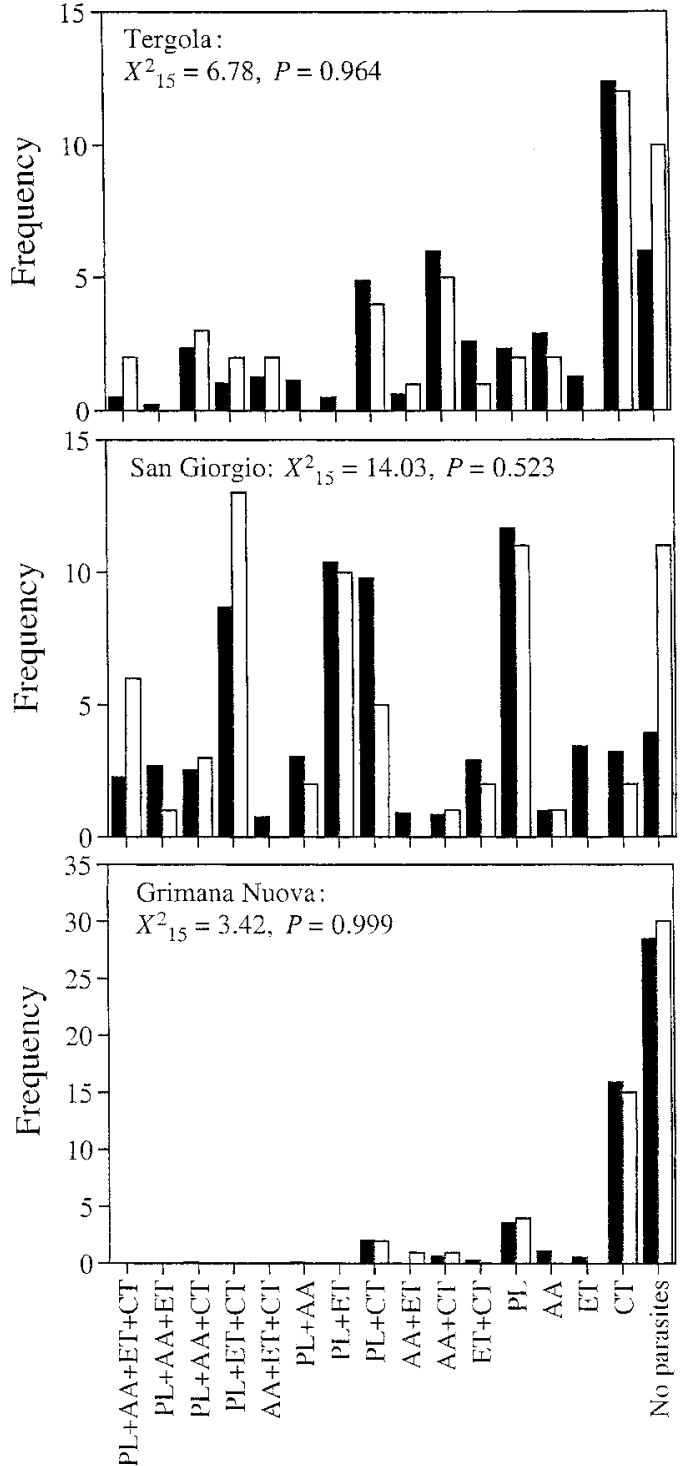

Fig. 1. Frequency (number of fish) distribution of different cooccurrences of four helminth species in three populations of trout, Salmo trutta, from northern Italy. The expected frequencies (black bars) of co-occurrences were compared with observed ones (open bars) using a Chi-square test. Key: PL, Pomphorhynchus laevis; AA, Acanthocephalus anguillae; ET, Echinorhynchus truttae; CT, Cyathocephalus truncatus.

interactions in structuring helminth communities (Poulin, 1998, 2001). Here we investigated species associations among helminths of brown trout, S. trutta, and found overall support for a role of negative, probably competitive interactions in shaping the helminth community. The co-occurrences of species, based on presence/ absence data, were not different from what would be expected by chance. This suggests that the negative interactions between helminth species are not strong enough to result in the exclusion of one species from hosts harbouring a second species. However, when using data on numbers of helminths per fish rather than merely their presence, we found consistently (though not always significant) negative correlations between pairs of helminth species. These negative relationships appeared to be stronger in pairs of helminths that used different intermediate hosts, but were nonetheless also found among pairs of helminths that share the same intermediate host. Since the size (or age) of the host can also be ruled out as a confounding factor, these results point toward a role of competition in helminth community structure.

We tried to exclude two potentially confounding variables that may either mask existing relationships or create spurious ones. First, variability in the size of fish hosts within a sample used for analyses of helminth community structure can create apparent associations that have nothing to do with interspecific interactions. Individuals of two different parasite species can accumulate simultaneously inside fish hosts as these grow, generating positive interspecific relationships in the process. The greater likelihood of certain species being found in larger fish can also influence community patterns (e.g. Guégan \& Hugueny, 1994). Although the effect of fish size on both the abundance and richness of helminth parasites is well documented, there are many reasons why this effect is not always noticeable (see Poulin, 2000). Whatever the reason, fish size had no apparent impact on helminth community structure in this study. Second, the transmission of larval helminths in packets from intermediate hosts to definitive hosts could lead to a transfer of associations (Lotz et al., 1995). We found no clear-cut evidence that pairs of helminth species sharing an intermediate hosts were more or less likely to show a significant association within the fish host than pairs of helminths using different intermediate hosts. In this system, the only pair of species for which data are available from intermediate hosts is Pomphorhynchus laevis and Acanthocephalus clavula: these two species co-occur more often than expected in their amphipod intermediate host (Dezfuli et al., 2000). Because only three fish from one stream harboured $A$. clavula, it was not possible to test for a direct transfer of the positive association from the amphipod host to the fish host.

A predominance of negative associations in communities dominated by acanthocephalans is not surprising in fish. There have been reports of one acanthocephalan species rendering the intestinal habitat unsuitable for other species, including some of the species investigated here (e.g. Thomas, 1964; Bates \& Kennedy, 1990; VidalMartinez \& Kennedy, 2000). Certain species, such as $P$. laevis, also compete intraspecifically for attachment sites along the intestinal wall (Brown, 1986). Some of the species found in brown trout are capable of adjusting their location within the intestine to minimize their interactions with other helminth species, at least in certain host species (Kennedy \& Moriarty, 1987; Bates \& Kennedy, 1990). Since it is the numerical effect of one species on another that has the greatest impact on population dynamics and that provides the best index of interspecific competition (Thomson, 1980), the generally negative relationships found here support the idea that competitive interactions are common in helminth communities. 
Table 3. Matrix of pairwise associations (Spearman's rank correlation coefficients) between the intensity of infection of the four helminth species commonly found in trout, Salmo trutta, from three streams in northern Italy. Fish not harbouring worms from either species in a pairwise association (double zeros) were excluded; actual sample sizes are the numbers of fish harbouring at least one of the two species in a pair, and are given below the diagonal.

\begin{tabular}{|c|c|c|c|c|}
\hline & P. laevis & A. anguillae & E. truttae & C. truncatus \\
\hline \multicolumn{5}{|l|}{ Tergola } \\
\hline Pomphorhynchus laevis & - & -0.260 & -0.119 & -0.118 \\
\hline Acanthocephalus anguillae & 23 & - & -0.367 & -0.192 \\
\hline Echinorhynchus truttae & 17 & 18 & - & -0.230 \\
\hline Cyathocephalus truncatus & 33 & 34 & 32 & - \\
\hline \multicolumn{5}{|l|}{ San Giorgio } \\
\hline Pomphorhynchus laevis & - & 0.083 & -0.100 & $-0.222^{*}$ \\
\hline Acanthocephalus anguillae & 53 & - & $-0.530^{* * *}$ & -0.150 \\
\hline Echinorhynchus truttae & 53 & 39 & - & $-0.276^{*}$ \\
\hline Cyathocephalus truncatus & 56 & 36 & 42 & - \\
\hline \multicolumn{5}{|l|}{ Grimana Nuova } \\
\hline Pomphorhynchus laevis & - & $-0.803^{* *}$ & $-0.676^{*}$ & $-0.535^{* *}$ \\
\hline Acanthocephalus anguillae & 8 & - & - & $-0.472^{* *}$ \\
\hline Echinorhynchus truttae & 7 & 2 & - & $-0.383^{*}$ \\
\hline Cyathocephalus truncatus & 23 & 20 & 20 & - \\
\hline
\end{tabular}

${ }^{*} P<0.10,{ }^{* *} P<0.05,{ }^{* * *} P<0.005$.

It must be pointed out, however, that none of the species pairs yielded a significant negative correlation in more than one stream. Typically, studies like the present one are only performed on a fish sample from a single locality. It is therefore quite possible that the results of earlier studies may not be consistent, or at least highly variable, across host populations. Prevalence and mean intensity of infection by the four common helminth species studied here varied across populations, and none of the four rare helminth species occurred in all three streams (table 1). Conditions therefore varied among the three trout populations, and competitive interactions between helminth species may be detectable under certain conditions but not in other conditions (e.g. Bates \& Kennedy, 1990, 1991). This point has been made by Dobson (1985), who used models to show that the abundance and dispersion of parasite species among host individuals influence the strength of interspecific competition. Thus without replication, it is impossible to conclude safely that any two helminth species always interact: results from a single host population are likely to reflect the local conditions only (see also Carney \& Dick, 2000). The main conclusion from our study, then, is that the repeatability of interspecific interactions in helminth communities is low, and that general interpretations are not valid unless derived from many host populations.

\section{Acknowledgements}

Financial support was provided by the Province of Padua and the Italian Ministry of University and Scientific Research and Technology.

\section{References}

Bates, R.M. \& Kennedy, C.R. (1990) Interactions between the acanthocephalans Pomphorhynchus laevis and Acanthocephalus anguillae in rainbow trout: testing an exclusion hypothesis. Parasitology 100, 435-444.

Bates, R.M. \& Kennedy, C.R. (1991) Potential interactions between Acanthocephalus anguillae and Pomphorhynchus laevis in their natural hosts chub, Leuciscus cephalus and the European eel, Anguilla anguilla. Parasitology 102, 289-297.

Brown, A.F. (1986) Evidence for density-dependent establishment and survival of Pomphorhynchus laevis (Müller, 1776) (Acanthocephala) in laboratory-infected Salmo gairdneri Richardson and its bearing on wild populations in Leuciscus cephalus (L.). Journal of Fish Biology 28, 659-669.

Bush, A.O. \& Holmes, J.C. (1986) Intestinal helminths of lesser scaup ducks: an interactive community. Canadian Journal of Zoology 64, 142-152.

Bush, A.O., Heard, R.W. \& Overstreet, R.M. (1993) Intermediate hosts as source communities. Canadian Journal of Zoology 71, 1358-1363.

Carney, J.P. \& Dick, T.A. (2000) Helminth communities of yellow perch (Perca flavescens (Mitchill)): determinants of pattern. Canadian Journal of Zoology 78, 538555.

Dezfuli, B.S. \& Scholz, T. (1995) Cyathocephalus truncatus (Cestoda: Spathebothridea) in its intermediate host Echinogammarus stammeri (Amphipoda) from the River Brenta, northern Italy. Parassitologia 37, 59-62.

Dezfuli, B.S., Rossetti, E., Fano, E.A. \& Rossi, R. (1994) Occurrence of larval Acanthocephalus anguillae (Acanthocephala) in Asellus aquaticus (Crustacea: Isopoda) from the River Brenta. Bollettino di Zoologia 61, 77-81.

Dezfuli, B.S., Rossetti, E., Bellettato, C.M. \& Maynard, B.J. (1999) Pomphorhynchus laevis in its intermediate host Echinogammarus stammeri in the River Brenta, Italy. Journal of Helminthology 73, 95-102. 
Dezfuli, B.S., Giari, L. \& Poulin, R. (2000) Species associations among larval helminths in an amphipod intermediate host. International Journal for Parasitology 30, 1143-1146.

Dobson, A.P. (1985) The population dynamics of competition between parasites. Parasitology 91, 317347.

Ellis, R.D., Pung, O.J. \& Richardson, D.J. (1999) Site selection by intestinal helminths of the Virginia opossum (Didelphis virginiana). Journal of Parasitology 85, 1-5.

Guégan, J.-F. \& Hugueny, B. (1994) A nested parasite species subset pattern in tropical fish: host as major determinant of parasite infracommunity structure. Oecologia 100, 184-189.

Haukisalmi, V. \& Henttonen, H. (1998) Analysing interspecific associations in parasites: alternative methods and effects of sampling heterogeneity. Oecologia 116, 565-574.

Holmes, J.C. (1961) Effects of concurrent infections on Hymenolepis diminuta (Cestoda) and Moniliformis dubius (Acanthocephala). I. General effects and comparison with crowding. Journal of Parasitology 47, 209-216.

Janovy, J. Jr., Clopton, R.E., Clopton, D.A., Snyder, S.D., Efting, A. \& Krebs, L. (1995) Species density distributions as null models for ecologically significant interactions of parasite species in an assemblage. Ecological Modelling 77, 189-196.

Kennedy, C.R. (1978) An analysis of the metazoan parasitocoenoses of brown trout Salmo trutta from British lakes. Journal of Fish Biology 13, 255-263.

Kennedy, C.R. \& Hartvigsen, R.A. (2000) Richness and diversity of intestinal metazoan communities in brown trout Salmo trutta compared to those of eels Anguilla anguilla in their European heartlands. Parasitology 121, $55-64$.

Kennedy, C.R. \& Moriarty, C. (1987) Co-existence of congeneric species of Acanthocephala: Acanthocephalus lucii and A. anguillae in eels Anguilla anguilla in Ireland. Parasitology 95, 301-310.
Lafferty, K.D., Thomas, F. \& Poulin, R. (2000) Evolution of host phenotype manipulation by parasites and its consequences. pp. 117-127 in Poulin, R., Morand, S. \& Skorping, A. (Eds) Evolutionary biology of host-parasite relationships: theory meets reality. Amsterdam, Elsevier Science.

Lotz, J.M. \& Font, W.F. (1994) Excess positive associations in communities of intestinal helminths of bats: a refined null hypothesis and a test of the facilitation hypothesis. Journal of Parasitology 80, 398-413.

Lotz, J.M., Bush, A.O. \& Font, W.F. (1995) Recruitmentdriven, spatially discontinuous communities: a null model for transferred patterns in target communities of intestinal helminths. Journal of Parasitology 81, 12-24.

Patrick, M.J. (1991) Distribution of enteric helminths in Glaucomys volans L. (Sciuridae): a test for competition. Ecology 72, 755-758.

Poulin, R. (1998) Evolutionary ecology of parasites: from individuals to communities. London, Chapman \& Hall.

Poulin, R. (2000) Variation in the intraspecific relationship between fish length and intensity of parasitic infection: biological and statistical causes. Journal of Fish Biology 56, 123-137.

Poulin, R. (2001) Interactions between species and the structure of helminth communities. Parasitology (in press in Suppl. to Vol. 122).

Thomas, J.D. (1964) Studies on populations of helminth parasites in brown trout (Salmo trutta L.). Journal of Animal Ecology 33, 83-95.

Thomson, J.D. (1980) Implications of different sorts of evidence for competition. American Naturalist 116, 719726.

Vidal-Martinez, V.M. \& Kennedy, C.R. (2000) Potential interactions between the intestinal helminths of the cichlid fish Cichlasoma synspilum from southeastern Mexico. Journal of Parasitology 86, 691-695.

(Accepted 22 January 2001)

(C) CAB International, 2001 